\title{
ANALYSIS OF ROOT DEVELOPMENT IN ARABIDOPSIS THALIANA
}

Ben Scheres. ${ }^{1 *}$, Viola Willemsen ${ }^{1}$, Kees Janmaat ${ }^{1}$, Harald Wolkenfelt ${ }^{1}$, Liam Dolan ${ }^{2}$ and Peter Weisbeek ${ }^{1}$.

1: Department of Molecular Cell Biology, University of Utrecht, Padualaan 8, $3584 \mathrm{CH}$ The Netherlands.

2: Department of Cell Biology, John Innes Institute, Colney Lane, Norwich, United Kingdom.

*: Corresponding author.

\section{Introduction}

Mature plants are the result of two formally separable developmental processes. Embryogenesis provides a mature embryo with axis, cotyledons, and the root and shoot apical meristem. The embryo has a structure of limited complexity which is relatively uniform in flowering plants. Upon seed germination post-embryonic development starts. The primary root and shoot meristems, together with newly formed secondary meristems, elaborate the architecture of the mature plant. We are interested in finding out how meristematic cells in the embryo are programmed to form organs with appropriately spaced tissues and cell types.

Meristem formation can be considered as a special case of cell specification according to position. Programming cells for a specific role in accordance with their position is a central issue in development of all multicellular organisms. The spatial organisation of specified cells, organised in tissues, is heritable and thus necessarily under some form of genetic control. The term 'pattern formation' refers to the ontogeny of this spatial arrangement of cells. Identification of genes involved in pattern formation in Drosophila has been an essential step towards our general understanding of regional specification mechanisms (e.g. Ingham, 1988). The applicability of genetic analysis to plant development is demonstrated by the progress in studies on flower development, initiated by the description of floral homeotic mutants (reviewed by Meyerowitz \& Coen, 1991). On these grounds we also favor the genetic approach to analyse cellular specification within meristems.

How is pattern formation at the cellular level achieved? The general picture that emerges from studies on Drosophila embryogenesis is one of a coarse regional specification by concentration gradients of gene products, mostly transcription factors. Combinatorial codes of these products define regions in the embryo (St Johnston \& Nüsslein-Volhard, 1992; Gaul and Jäckle, 1989; Hülskamp et al, 1990). This definition of spatial regions is subsequently refined to ultimately yield cellular specification at appropriate positions. An important role for cell-cell signalling in the final stages of cellular specification is not only evident from studying Drosophila embryogenesis (Ingham and Martinez Arias, 1992), but also from studies on Drosophila eye development and vulva formation in Caenorhabditis elegans (Rubin, 1991; Horvitz \& Sternberg, 1991). The concept of coarse positional information which is gradually refined to the level of cellular interactions may well be of general importance. 
In plants, genes involved in regional specification have also been identified. During Arabidopsis embryogenesis a set of zygotically active genes are postulated to define the main regions of the embryo, e.g. shoot meristem, cotyledons, hypocotyl, and root (Mayer et al, 1991). At the postembryonic stage of plant development, genes involved in primordium specification in flowers have been identified and analyzed at the molecular level (reviewed by Meyerowitz \& Coen, 1991). Noteworthy combinatorial codes are proposed to be important for regional specification in both these studies, analogous to the mechanisms unraveled in Drosophila. Genes which regulate pattern formation at the cellular level have not been analysed systematically in plants. It is our aim to identify such genes, involved in programming meristematic cells.

Root meristems produce cell files which can be traced back to meristem initial cells. This simplifies the explanation of an observed pattern defect in terms of altered activity of meristematic cells. On the other hand, clonal analysis of the Arabidopsis shoot meristem indicated that only a loose association between meristematic cells and differentiated cells exists (Irish \& Sussex, 1992; Furner \& Pumfrey, 1992). Hence pattern defects in tissues derived from the shoot meristem will not always be relatable to defects in specific meristematic cells. In order to be able to take our study to the cellular level we therefore chose to analyse the root meristem of Arabidopsis, a plant well suited for genetic analysis (Meyerowitz, 1989). In this paper we will first describe the anatomy of the Arabidopsis root including the arrangement of the meristematic initial cells. Subsequently the embryonic origin of the root and the proposed meristematic initials will be elaborated on, referring to both anatomical studies and clonal analysis data. Finally we will present the results of a genetic screen designed to pinpoint genes involved in pattern formation within the root meristem. We will discuss these results within the conceptual framework of gradually refined positional information as sketched above.

\section{Descriptive analysis of root development}

Exact knowledge on the position of specific cell types and their clonal origin greatly adds to the interpretation of mutant phenotypes, and is an indispensable prerequisite for detailed genetic analysis of pattern formation at the cellular level. This is impressively demonstrated by the follow-up research leaning on the description of the Caenorhabditis elegans cell lineage (Sulston et al, 1983; Stemberg and Horvitz, 1984; Horvitz \& Sternberg, 1991).

\section{Meristematic cells in the ARABIDOPSIS root.}

Anatomical analysis reveals a simple and constant organization of the mature Arabidopsis root. Only single layers of each of the cell types outside the central cylinder are present, and the numbers of cells in these layers and inside the central cylinder are relatively invariant. On the outside of the root one layer of epidermal cells is present comprising on average 19 cells. Depending on the distance from the tip one or more lateral root cap layers (32 cells on average) can be distinguished, overlying the epidermal cell layer. Epidermal cells can be divided in roothair forming cells (trichoblasts) and non-root hair forming cells which are arranged in a regular and predictable fashion. To the inside of the epidermis one layer of 8 cortex cells and one layer of 8 endodermis cells is present. Each cortex cell is neighbouring one endodermal cell. Underneath the endodermis the outer layer of the central cylinder, consisting of on average 12 pericycle cells, is situated. The remaining cells form the central cylinder and contain the 
vascular cells which show a diarch organisation with two protoxylem and two protophloem poles.

The origin of cell files that form the mature root tissue can be inferred from median longitudinal sections of root tips. The Arabidopsis root contains separate tiers of cells from which the different tissue layers originate, to be called 'initials' hereafter. Such an organization of the root meristem is referred to as the 'closed meristem' type (Clowes, 1981). Epidermis and lateral root cap share common initial cells. Cortex and endodermis also share initial cells. A prepatterned set of initial cells is present for cells inside the vascular cylinder. At the distal end of the root each of the twelve cell files of the columella part of the root cap has its own initial. Four 'central' cells are laterally continuous with the cortex and endodermis cells. These cells do not need to divide to preserve the observed structural integrity of the root. In vivo ${ }^{3} \mathrm{H}$ Thymidine incorporation studies indicate that these cells have a much slower cell cycle than the surrounding cells, and therefore we regard them as 'quiescent centre' cells (Clowes, 1956). Noteworthy, these cells are facing all the cells which we propose to act as initials for the different root tissues.

Anatomical arguments alone can not prove that the cells we propose to be initials truly participate in pattern formation. However, ${ }^{3} \mathrm{H}$-Thymidine incorporation was clearly observed in all these cells, indicating that they divide actively. In addition, mitotic figures can be found in the cells predicted to be initials. Derivatives of the initial cells in the meristematic zone appear to have a more rapid cell cycle time than the initials themselves. The derivatives of the columella initials are an exception in that they do not divide again.

Transverse sections of several root tip regions containing the initial cells invariantly depict 16 epidermal initials, 8 cortex/endodermal initials, 9-10 pericycle initials, and 12 columella initials. We take this as an indication that the relative invariance in cell numbers of the mature root finds its origin in even more stringently fixed numbers of initials.

In summary, the developmental history of the Arabidopsis root can be traced back to the activity of a fixed number of initials surrounding a small quiescent centre. It must be noted that an eventual ability of the cells in the quiescent centre to participate in pattern formation under certain specific conditions cannot be ruled out by our experiments. Such activity has been proposed for quiescent centre cells and a comparison to animal stem cells has been made (Barlow, 1978). We have observed alterations in cell shape and division planes in the meristematic area in ageing roots. Nevertheless we have not been able so far to obtain clear evidence for division of the central cells.

\section{Embryonic origin of the root meristem initials}

The tiered organisation of the Arabidopsis root meristem initials puts forth the question to the origin of these cells. Transverse sections from the root tip of imbibed seeds prior to germination demonstrate that the complete setting of rings and plates of initials, surrounding the four central quiescent cells, is alreadly apparent in the mature embryo. It is therefore not established during post-embryogenic development from a simpler version of the root meristem. The numbers of different initials in the mature embryo equal the numbers found in the active root meristem of the seedling. In order to investigate how these initials, which constitute the root meristematic core, come about during embryogenesis we have performed anatomical studies of embryos at 
successive developmental stages. The development of the root apex has been described in several Brassicaceae including Arabidopsis (von Guttenberg, 1947; Tyrkaska, 1979; Mansfield \& Briarty, 1991). We have extended these studies to understand the exact patterning of root meristem initials during embryogenesis.

Median longitudinal sections at the mature embryo stage reveal an organisation of the root meristem very similar to what is observed in seedlings. However, fewer layers of both columella and lateral root cap are present. The same coordinate divisions as proposed for the post-embryonic activity of the root meristem initials can explain the ontogeny of the embryonic root (radicle). Sections of earlier stages of embryogenesis are consistent with the view that this sequence of divisions first starts at the late heart stage, and is anatomically marked by the appearance of a periclinal division in epidermal cells that give rise to the first layer of lateral root cap in the embryo. In this view the structure of initials, surrounding the quiescent centre cells, is defined in mid-heart stage embryos just before this characteristic division is apparent.

A next question which is important is the clonal origin of the different initials that appear specified at mid-heart stage. Sections of globular stage embryos indicate that the central 'quiescent' cells, and the columella initials are derivatives of the hypophyseal suspensor cell. The hypophysis in turn originates from divisions of the basal cell after the first zygotic cleavage (for a description and definition of embryonic stages see Jürgens and Mayer, 1993). In contrast, all the other initial cells derive from the apical cell that results from the first zygotic cleavage. Henceforth initials of the root meristem appear to have different clonal origin.

Analysis of the numbers of cells in the different tiers of initials shows that the number of epidermal and cortical initials is already established at the globular stage of embryogenesis. The numerical invariance of these sets of initials therefore appears to be a consequence of the rigid control of cell division at that stage. Cell numbers in the vascular cylinder are established much later in embryogenesis. Nevertheless the pattern of vascular initials, like the pattern of epidermis and cortex, is a result of radial pattern formation in the complete embryonic axis which will give rise to both hypocotyl and root.

\section{The embryonic root: not entirely derived from meristem initials?}

In the mature embryo the region of the root which is formed by the activity of the meristem initials is marked by the presence of the lateral root cap. On the other hand the hypocotyl region of the mature embryo is marked by the presence of a second cortex cell layer, not present in the root. Furthermore the hypocotyl region contains more epidermal cells in circumference.

Noteworthy, a region in the mature embryo in between the meristematic root and the hypocotyl is characterized by the presence of either one cortex cell layer, or a second, inner, cortex cell layer that consists of wedge-shaped cells. This layer is not derived from the meristematic initials as no lateral root cap is present. At the late heart stage this 'intermediate' layer can be assumed as the region with the height of about three epidermal cell tiers immediately above the initials which already gave rise to the lateral root cap.

At the seedling stage the connection between root and hypocotyl reveals a region which shares root and hypocotyl characteristics. The inner cortex cells in this region are wedge-shaped and 
the number of epidermal cells in circumference increases more proximal to the root tip. Therefore this region acquires more 'hypocotyl characteristics' when the boundary with the true hypocotyl, which has a uniform radius, is approached. However, the presence of root hairs defines the region as part of the root in terms of cellular differentiation. This zone, which we call 'interzone' hereafter, can be presumed to arise from the previously mentioned intermediate layer in the embryo. In summary, anatomical data suggest that the root meristem initials are not the only progenitors of the root.

\section{Clona1 analysis of the ARABIDOPSIS root using transposon excision}

In the previous paragraph two embryonic regions involved in root formation were distinguished. One which gives rise to the tier of initials derived from the apical zygotic cell. A second layer of cells, defined at the late heart stage, was proposed to form a region of the root not elaborated by the initials. This assumption cannot be proven with anatomical studies alone. An alternative scenario may be proposed in which cortical cells within the intermediate embryonic layer undergo further periclinal divisions. In this way the complete intermediate region could be incorporated in the 'true' hypocotyl of the seedling. Such a model predicts that the complete root derives from the meristematic initials. In order to test which of these alternative developmental scenarios is happening, we performed clonal analysis.

For this purpose transgenic plants, kindly provided by Emily Lawson and Carolyn Dean (John Innes Inst., U.K.), were used. These plants contain a T-DNA insert containing a $35 \mathrm{~S}$ CaMV promoter, a maize Ac element, and B-glucuronidase (GUS) coding sequences. Upon excision of the Ac element in embryonic cells, clones of descendant cells can be formed in which GUS activity can be histologically detected. Embryonic clonal sectors were sampled from appr. 100,000 Xgluc-stained seedlings derived from a transgenic line with moderate excision frequency. This particular line was chosen because excision frequency was high enough to collect embryonic sectors, and low enough to avoid frequent sector overlap.

Sectors spanning the complete root, interzone, hypocotyl, or a combination of these regions were analyzed. All sectors spanning the complete hypocotyl ended at a sharp lower boundary where the hypocotyl was ending and the root hair containing epidermis cells became first evident ('lower' is referring to positions closest to the root apex, and 'upper' to positions closest to the shoot apex). Sectors spanning the complete root were found and they had their upper boundaries at the same position mentioned above where the first root hairs become apparent. We took this as an indication that root and hypocotyl arise from two embryonic tiers in which at least epidermal cells, after the separation of these tiers, have very limited potential to switch root or hypocotyl fate. The next-largest root sectors found with similar boundaries fall in two classes: interzone sectors in which the lower sector boundary coincides approximately with the region in the root where the interzone-specific second cortex layer disappears; and root sectors originating from the meristem and having their upper sector boundary in the same region.

Currently we are saturating this data set. So far, the sector analysis is consistent with the proposition that three distinct embryonic tiers give rise to hypocotyl, interzone, and initials. In this view the interzone derives from the 'intermediate' cell tiers apparent in the embryo. The remaining basal portion of the root in the seedling appears to derive from the meristematic initials. 


\section{Identifying 'root meristem formation genes': rationale}

From the anatomical and clonal analyses on the Arabidopsis root we draw the following main conclusions. First, root meristem initials appear specified at the late heart stage of embryogenesis. Second, the radial pattern of root meristem initials, and henceforth the radial anatomy of the root, is a reflection of the divisions which establish the radial arrangement of cells in the complete embryonic axis. Third, the Arabidopsis root consists of a region which is derived from the meristematic initials, and an interzone region at the basis of the hypocotyl which exhibitis root-specific differentiation but appears to derive from a seperate embryonic cell layer.

These three features have consequences for mutant phenotypes which are expected to define genes involved in the specification of the root meristematic cells. First, defective specification of the meristematic initials is expected to yield deviations in embryo development from late heart stage onward. Second, radial pattern mutations evident in the root can be expected to have an embryonic origin. Third, mutations affecting root meristem formation may not be completely rootless, since the interzone can differentiate to root specific cell types.

Bearing these consequences in mind we performed a screen for mutants defective in root meristem formation. Since the majority of the mutantions we were interested in would be expected to yield embryonic phenotypes we anticipated that many mutants could be seedlinglethal. Therefore we performed a screen using single siliques from 10,000 separate $F 1$ families, to be tested for root meristem mutations in the F2 generation. In this way heterozygous plants carrying mutant alleles can be rescued (Jürgens et al, 1991). From this screen we collected all families that segregated phenotypes with an affected root. We reasoned that we could not on forehand anticipate the severity of phenotypes associated with meristematic defects. This bears on the simple radial anatomy of the root, which is an advantage in interpreting the cause of a particular phenotype, but a disadvantage in finding phenotypes. Therefore we considered every family showing a root defect for a somewhat more detailed analysis using 'whole mount' preparations of cleared roots.

\section{Mutations perturbing root meristem development}

141 F1 lines from our screen segregated mutants in a Mendelian fashion with abnormalities in root growth or development throughout two subsequent generations. These lines were grouped in classes ranging from severe to mild distortions of root development. Below we will briefly describe three classes of mutants which we believe to be significant in the light of the description of wildtype development as discussed above. These classes of mutants are not covering all different root mutants which we have detected and which are currently under analysis. For example, radial pattern mutants and shape mutants have been found but they often exhibit pleiotropic effects which hampers interpretation of the phenotype. 


\section{'Early' embryonic pattern mutants}

Seedlings with severely affected development of the basal region, and hence the root, have been found in our screen and they fully fit the phenotypic description of plants carrying gnom and monopteros alleles, described by Mayer et al (1991). The majority of gnom seedlings lack differentiation of the root. Mutation in the gnom gene have been shown to already disturb the first assymetric division of the zygote, but may also be involved in assymetric divisions defining the hypophyseal suspensor cell (Mayer et al, 1993). Mutations in the monopteros gene can lead to the complete absence of root and hypocotyl. The phenotype can be traced back to the octant stage of embryogenesis where supernumerary cell tiers are present in the embryo proper (Berleth \& Jürgens, in press). The cell in monopteros mutant embryos at the position of the hypophyseal cell in wildtype embryos appears to be only able to perform transverse divisions.

In conclusion, both gnom and monopteros genes are involved in defining the basal region of the embryo at early stages of development. Their function is a prerequisite for setting up the conditions for correct development of the root meristem.

\section{'Heart stage' embryonic pattern mutants}

We identified several lines segregating mutant phenotypes that lack the characteristic anatomical features of the root meristematic area. Noteworthy, several tiers of cells at the basal end of the hypocotyl of mutant seedlings bear root hairs and show the decrease in radial diameter characteristic for the interzone. Therefore the embryonic root appears to have developed normally.

An example of this class of mutants is hobbit. Using Nomarsky optics on whole mounts of hobbit embryos deviations from the typical division sequences of the initials can clearly be observed at the heart stage. At that stage no clear alterations in the apical part of the embryo can be observed. At seedling stage, however, it becomes evident that the presence of the mutation leads to smaller seedlings with altered cotyledon shape. The shoot meristem produces leaves but we have not been able so far to induce flowering. In contrast, another member of this class lacking of a patterned root meristem, orc, exhibits no shoot meristem activity.

\section{Meristem activation mutants}

Mutants of this class produce very short roots and normal, mostly fertile shoots. This indicates very limited or no activity of root meristematic cells. The root tip contains the typical setting of initials surrounding a quiescent centre. However, division patterns of initial cells which apparently could be performed during embryogenesis appear are not perpetuated at the postembryonic stage. An example of this class of mutants is elf. The primary root remains inactive while the hypocotyl elongates normally. Normal sized cotyledons are present at seedling stage, and a morphologically normal albeit sterile shoot it made. After several weeks on sucrose containing medium, lateral roots are formed with normal appearance. This indicates that the lack of meristem activation is specific for the primary meristem. In contrast, other members of this class initiate lateral roots which are, like the primary root, unable to perpetuate meristematic activity. 


\section{Concluding remarks}

Anatomical analysis of the Arabidopsis root reveals an elegantly simple and highly ordered arrangement of different cell types. Meristematic initials can be identified, and their active participation in pattern formation is inferred from in vivo ${ }^{3} \mathrm{H}$-Thymidine labeling experiments. A 'core meristem' has been pinpointed, containing a small centre of quiescent cells surrounded by initials for all the different root tissues, and the embryonic origin of this core meristem is elucidated. This description of root development at the cellular level seems to make the Arabidopsis root ideally suited for genetic dissection of root meristem formation and function. One can question whether the genetic determinants of root development of the tiered meristem type, as in Arabidopsis, are significant for understanding the activity of untiered, 'open' root meristems (Clowes, 1981). Initials in open meristems face developmental decisions leading to more different cell types. However, epidermal initials in the Arabidopsis root have to produce sister, epidermis and lateral root cap cells. Likewise, cortex initials have to produce sister, endodermal and cortical cells. We can envisage molecular mechanisms underlying these developmental decisions identical to the ones that direct initials of untiered meristems to produce more different cell types.

Furthermore one can wonder whether understanding the formation of a root with a rather strict lineage-dependent meristematic activity has any relevance for understanding shoot development. In this context it is worthwile to mention that the development of the worm

C. elegans, a nematode with invariant cell lineages, involves many cellular interactions. Likewise, the development of the Arabidopsis root may be continuously relying on positional cues to a greater extent than expected from the cell lineage relations (Barlow, 1984). If this is the case overlapping mechanisms for root and shoot meristem activity may exist, since for shoot development it is clear that positional information is important (e.g. Sussex, 1989).

The classes of mutations that we have found, clearly show that formation of a functional root meristem can be blocked at several stages. We envisage a gradual process in which first the basal part of the seedling is defined, depending on the products of genes like gnom and monopteros. This specification can then be refined by products of genes like hobbit, ultimately leading to cellular delimitation of the cells of the core meristem. Finally, upon germination, genes defined by the meristem activation mutations enable perpetuation of cell division by the initials and their derivatives.

As in the case of Drosophila, these propositions will have to be substantiated by both genetic and molecular analysis. Extending the analogy to animal systems, it will be interesting to see whether we have to envisage the broad specification genes as transcription factors, and the ones involved in cellular specification as products involved in communication between cells.

During the spatial definition of the root meristem in the apical-basal axis, the radial pattern must also be defined. We have shown that the radial pattern, set up early in embryogenesis, defines the setting and the numbers of root meristem initials, and hence is directly related to the radial anatomy of the root. Therefore we envisage the radial anatomy of the root as an embryonic property. In a collaboration with L. Di Laurenzio and P. Benfey (New York University) we showed that the shortroot mutation, which lacks an endodermis in the root, results from a radial pattern defect during embryogenenis which also penetrates into the hypocotyl. This is in accordance with our view that mutant phenotypes with altered radial pattern in the root may generally be traced back to embryogenesis. 


\section{References}

Barlow,P.W. (1978). The concept of the stem cell in the context of plant growth and development. In: stem cells and tissue homeostasis, B.I. Lord, C.S. Potten \& R.J. Cole, eds. Cambridge University Press, 87-113.

Barlow,P.W. (1984). Positional controls in root development. In: positional controls in plant development. P.W. Barlow \& D.J. Carr, eds. Cambridge University Press.

Berleth,T. and Jürgens,G. (1993). The role of the monopteros gene in organising the basal body region of the Arabidopsis embryo. Development 118 (in press).

Clowes,F.A.L. (1956). The cytogenerative centre in roots with broad columellas. New Phytol. 48-57.

Clowes,F.A.L. (1981). The difference between open and closed meristems. Ann. Bot. 48, 761767.

Furner,I.J., and Pumfrey,J.E. (1992). Cell fate in the shoot apical meristem of Arabidopsis thaliana. Development 115, 755-764.

Gaul,U., and Jäckle,H. (1989). Analysis of maternal effect mutant combinations elucidates regulation and function of the overlap of hunchback and Krüppel gene expresssion in the Drosophila blastoderm embryo. Development 107, 651-662.

Guttenberg, von,H. (1947). Studien über die Entwicklung des Wurzelvegetationspunktes der Dikotyledonen. Planta 35, 360-396.

Horvitz,R.H. and Stemberg,P.W. (1991). Multiple intercellular signalling systems control the development of the Caenorhabditis elegans vulva. Nature 351, 535-541.

Hülskamp,M., Pfeifle,C., and Tautz,D. (1990). A morphogenetic gradient of hunchback protein organises the expression of the gap genes Krüppel and knirps in the early Drosophila embryo. Nature 346, 577-580.

Ingham,P.W. (1988). The molecular genetics of embryonic pattern formation in Drosophila. Nature 335, 25-34.

Ingham,P.W., and Martinez Arias,A. (1992). Boundaries and fields in early embryos. Cell 68, 221-235.

Irish,V.F., and Sussex,I.M. (1992). A fate map of the Arabidopsis embryonic shoot apical meristem. Development 115, 745-753.

Jürgens,G., Mayer,U., Torres Ruiz,R.A., Berleth,T., Miséra,S. (1991). Genetic analysis of pattern formation in the Arabidopsis embryo. Development 91 (Suppl. 1), 27-38.

Jürgens,G., and Mayer,U. (1993). Arabidopsis. In: a colour atlas of developming embryos. J. Bard (ed.), Wolfe Publishing, London.

Mansfield,S.G. and Briarty,L.G. (1991). Early embryogenesis in Arabidopsis thaliana. II. The developing embryo. Can. J. Bot. 69, 461-476.

Mayer,U., Torres Ruiz,R.A., Berleth,T., Misera,S., and Jürgens,G. (1991). Mutations affecting body organization in the Arabidopsis embryo. Nature 353, 402-407.

Mayer,U., Büttner,G. and Jürgens,G. (1993). Apical-basal pattern formation in the Arabidopsis embryo: studies on the role of the gnom gene. Development 117, 149-162.

Meyerowitz,E.M. (1989). Arabidopsis, a useful weed. Cell 56, 263-269.

Meyerowitz,E.M. and Coen,E.S. (1991). The war of the whorls: genetic interactions controlling flower development. Nature 353, 31-37.

Rubin,G.M. (1991). Signal transduction and the fate of the R7 photoreceptor in Drosophila. TIG 7, 372-377.

Sternberg,P.W., and Horvitz,H.R. (1984). The genetic control of cell lineage in nematode development. Ann. Rev. Genet. 18, 489-524. 
St. Johnston,D. and Nüsslein-Volhard,C. (1992). The origin of pattern and polarity in the Drosophila embryo. Cell 68, 201-219.

Sulston,J.E., Shierenberg,E., White,J.G., and Thomsom,J.N. (1983). The embryonic cell lineage of the nematode Caenorhabditis elegans. Dev. Biol. 100, 64-119.

Sussex,I.M. (1989). Developmental programming of the shoot meristem. Cell 56, 225-229.

Tyrkarska,T. (1979). Rape embryogenesis. II. Development of the embryo proper. Acta Socoetatis Botanicorum Poloniae 48, 391-421. 